\title{
Low-Level Laser Therapy for Herpesvirus Infections: A Narrative Literature Review
}

\author{
Sergey Vladimirovich Moskvin \\ 'O.K. Skobelkin State Scientific Center of Laser Medicine Under the Federal Medical Biological Agency, \\ Studencheskaya Str., 40, Moscow 121165, Russia
}

\section{*Correspondence to} Sergey Vladimirovich Moskvin Tel/Fax +7 (916) 987-9095 Email: 7652612@mail.ru

Received: January 20, 2021 Accepted: April 6, 2021 Published online July 24, 2021

\begin{abstract}
Introduction: Herpesvirus infection has a variety of clinical forms and is extremely widespread in the world while existing treatment methods are not always quite effective. The search for new treatment modalities is a relevant problem and numerous studies show the therapeutic effect of lowlevel laser therapy (LLLT) on different herpesvirus types.

Methods: The mechanisms of laser light action and the impact of LLLT on the pathological pathways of herpes infections are described. A narrative review of the relevant papers is conducted.

Results: The reviewed studies confirm that LLLT is a potential prospective treatment method for patients infected with the herpesvirus. However, it is necessary to improve the methodology and optimize the combination of laser action with antiviral medications.

Conclusion: The review shows that it is most effective to combine laser impact on skin lesions with the application of topical antiviral gels or creams, additionally using a combined procedure of laser ultraviolet blood illumination (LUVBI, 365-405 nm) + intravenous laser blood irradiation (ILBI, 525 nm).

Keywords: Herpesvirus infection; Urogenital tract; Low-level laser therapy.
\end{abstract}

\section{Introduction}

Among more than a hundred currently known herpesvirus types (Herpesviridae) only eight of them can affect humans ${ }^{1,2}$ :

- Alpha-Herpesviridae: Herpes simplex 1 (HSV-1), Herpes simplex 2 (HSV-2), chickenpox (HSV-3), or Varicella Zoster (VZV);

- Beta-Herpesviridae: Cytomegalovirus (CMV, HSV5), Herpes human virus 6 (HHV-6), Herpes human virus 7 (HHV-7);

- Gamma-Herpesviridae: Epstein-Barr (EBV, HSV-4) and HHV-8.

According to the World Health Organization data, nearly $90 \%$ of the world's population has one or more herpesvirus infections. Generally, patients are primarily infected in childhood, followed by subsequent latency development, which is characterized by the cessation of viral replication and viral protein expression. Reactivation of latent herpesviruses occurs due to the transient (infection, environmental factors, psycho-emotional stresses, endocrine disorders, etc.) or permanent (primary and secondary immunodeficiencies, immunosuppressive therapy) immunodeficiency conditions. Doctors in various fields of medicine manage the diseases caused by HSV-1, HSV-2 and HSV-3 viruses (chickenpox, shingles) using standardized diagnostics and treatment methods while the questions regarding their efficacy remain unsolved. To date, no clear algorithms or standards have been developed for the treatment of $\beta$ - and $\gamma$-herpesviruses, and this entails a number of problems with their clinical management. ${ }^{3}$

The pathogenesis of herpesvirus infection (HVI) is highly complicated and not well-established; the key points are that the acquired immunity is not developed and the mechanisms of the immune response to herpesvirus infection are highly multifactorial, with multi-level interactions between immunocompetent cells, cytokines, receptors, and so on. ${ }^{1}$

Viral cervicitis and vaginitis are the common causes of frequent visits to the physicians, with complaints about itching, burning sensations and profuse vaginal discharges. In the majority of males, CMV-infection (CMVI) of the reproductive system has no clinical signs, reflecting the primarily asymptomatic course of the disease. Available literature data demonstrate that the most severe cases of CMVI were observed in females, and the unsolved problems in their treatment efficacy still remain in focus. Regardless of the potential therapeutic arsenal of tools, it does not ensure the achievement of stable remission in all cases. ${ }^{4}$ 
Currently, acyclic nucleosides are the most wellestablished and efficient drugs for the treatment of herpesvirus infections. Acyclovir inhibits the synthesis of viral DNA immediately after phosphorylation and conversion to the active form - acyclovir triphosphate. Acyclovir triphosphate competitively inhibits viral DNA polymerase and, being a nucleoside analog, incorporates into viral DNA. This leads to obligate chain breaking, DNA-synthesis discontinuation, and virus replication termination. However, the bioavailability of acyclovir is $30 \%$, while bioavailability of valacyclovir and famciclovir is much higher $-54 \%$ and $77 \%$ respectively. Approved labelling for acyclovir, valacyclovir and famciclovir describes their selective activity in vitro against HSV, varicella zoster, Epstein-Barr, CMV, and additionally against HHV-6 for valacyclovir. ${ }^{3}$ The use of interferonand immunoglobulin-based drugs is the basis of the current immunotherapy. Although immunotherapy cannot replace antiviral chemotherapy completely, the additional use of the immunotherapeutic drugs allows improving treatment efficacy, reducing treatment duration and preventing the induction of resistance. ${ }^{5,6}$

Innovative treatment modalities, including low-level laser therapy (LLLT) for patients with HVI, have been developed in Russia and also abroad, where investigative attention to the low-intensity laser illumination (LILI) therapeutic effects is traditionally higher.

The purpose of the present article is to justify the use of LLLT for herpesvirus infections and to make recommendations for the treatment methods based on the literature review and our own data.

\section{Mechanisms of Laser Light Action}

The vast majority of reports confirm therapeutic effects of LILI on HSV-1, HSV-2 and varicella zoster infections as well as on postherpetic neuralgia. LLLT shortens the symptomatic period, alleviates pain and itching, reduces the rate and duration of recurrence, increases the duration of remission, and reduces the rate and intensity of postherpetic neuralgia. However, evidence regarding the direct LILI effects on viruses has not yet been obtained, at least for red and infrared ranges (633-1064 nm). The clinical effect, most likely mediated, is caused by both the activation of the sanogenetic processes in the patient's body and immune system modulation.

LLLT has an impact on all pathological pathways of the herpesvirus infection; it brings down inflammation, restores microcirculation, improves tissue metabolism disorders, and provides analgesic and other effects. Because of this, LLLT can replace a range of medications or enhance their actions. Importantly, laser illumination simultaneously induces inhibition of alteration (either primary or secondary at different stages of the disease) and activation of proliferation, with anti-exudative effect, which, taken together, provides the stimulation of regenerative processes and prevention of scarring (particularly with chronic ulcerative and ulcerativenecrotic herpesvirus lesions). Laser-induced analgesia significantly reduces sensations of irritation, itching, burning, swelling and others. Of special note is that laser illumination impacts on the local and systemic immune host defense mechanisms. A number of the performed pivotal experimental and clinical-laboratory studies elicited the highly effective mechanism of antiviral action. LILI stimulates the cell organelles functions (mitochondria, lysosomes, ribosomes) and significantly improves the cell resistance to pathogens, including viruses. In the presence of high cell resistance, even when penetrating the cell membrane, herpesvirus is not able to reprogram the functions of organelle cells and make the cell work for itself; as a result, its virulence is inhibited, and the process becomes abortive. With regard to prevention and treatment aspects, immunomodulatory properties of LILI, resulting in diminished sensitization, allergic reactions and immunodeficiencies, are of essential value. In addition, LILI demonstrates both the local (tissue) and general (health-promotion) effects, which is highly important in complicated cases, associated with the regulatory systems disorders and the inner organs pathology. ${ }^{7}$

Herpesvirus infections are often regarded by ophthalmologists, dentists, neurologists, pediatricians and other specialists as complications of the disease in their fields. In most cases, these infections are not considered to be a systemic problem requiring a comprehensive management approach. Generally, efforts are only focused on the elimination of local signs of viral activity. Such an approach nearly always results in a temporary effect, with the LLLT potential not completely realized.

\section{Narrative Literature Review}

When reviewing relevant studies on the subject published 40 years ago, one may note that every other article focuses on herpetic stomatitis. ${ }^{8}$ Nevertheless, the use of the local laser illumination method never became common due to its relatively low efficacy, although it is included in clinical recommendations in many countries worldwide. ${ }^{9}$ A lack of understanding of the core of LLLT methodology prevents foreign colleagues from implementing its potential in full. In addition, the efficacy of therapy, after all, means prolonged results, lasting for several years without chronic disease recurrence, rather than temporary results (successful relief of symptoms).

The herpetic keratitis proportion among all inflammatory corneal diseases is up to $80 \%$. Shakarian ${ }^{10}$ studied children with previously failed conventional therapies - in total, 27 patients, aged 4 to 10 years, with recurrent (17 patients) and primary (10 patients) conditions. Previous treatment duration ranged from 25 days to 1.5-2.0 months. In all cases, considerably reduced 
visual acuity, corneal opacification of various intensities with corneal staining of various shapes and areas, and moderate to severe irritation were observed at baseline. At the start of the LLLT, the status of the affected eye was evaluated in 10 patients as it worsened from the disease onset. The illumination was performed using continuous LILI in the red spectrum (wavelength of $633 \mathrm{~nm}$ ) over the courses of four to six procedures lasting 20 to 40 seconds, every other day, both directly on the ulcerated areas and the adjacent areas, as well as on the sites with neovascularization. The treatment cycle included one or two courses. After the first treatment course, improvement was observed in 20 patients. Corneal staining was preserved in three patients. Decreased opacification intensity was observed in eight patients. Therefore, adding the LLLT to the comprehensive conventional treatment for herpetiform keratitis in pediatric patients was proven to improve treatment efficacy considerably in a relatively short period (from 12 to 16 days). ${ }^{10}$

It was estimated that the combined LLLT method improved the treatment efficacy statistically significantly for all the clinical entities. This was primarily reflected in significant shortening (by 1.5-3 times) in the treatment duration and patient disability time. In the main group, there was complete clinical recovery in $82 \%$ and partial effect in $18 \%$; in the control group, complete recovery was shown only in $53 \%$ and partial effect in $36 \%$. It should also be stressed that $15 \%$ of the patients in the control group (with conventional therapy) had complications such as herpetic conjunctivitis converting into keratitis and kerato-iridocyclitis, and in the presence of herpetic kerato-iridocyclitis, more serious complications such as secondary glaucoma and epithelial-endothelial corneal dystrophy. No complications were observed in the main group. The analysis of a four-year follow-up demonstrated that the occurrence of the relapses was significantly reduced (by approximately $2-3.5$ times) in all studied clinical entities.

In Russia, LLLT has long been used successfully to treat patients with herpetic stomatitis, including pediatric populations. Reduced treatment duration, as well as a reduced number of relapses in children with chronic herpetic stomatitis, has been observed when using local illumination with continuous LILI (wavelength $633 \mathrm{~nm}$ ) on at least five lesions with an exposure time of one minute per lesion, with treatment courses consisting of from five to seven daily procedures, ${ }^{11}$ as well as pulsed infrared (IR) LILI. ${ }^{12}$ Foreign colleagues more often report on numerous cases of the clinical effectiveness of LLLT for herpes simplex, ${ }^{13-16}$ although, in addition to clinical studies, there are some isolated experimental works. For example, a study involving a model with HSV-1inoculated mice has demonstrated that illumination by IR LILI on the area C2-C3 prevented virus latency establishment. ${ }^{17}$ Results from another study on rabbits revealed herpesvirus inactivation due to illumination by LILI with $980 \mathrm{~nm}$ and $10600 \mathrm{~nm}$ wavelengths. ${ }^{18}$ An investigation into the impact of LILI, with parameters often used in the treatment of herpes labialis (wavelength $660 \mathrm{~nm}$ ), on DNA in the experimental model based on E. coli cell cultures and plasmids revealed that laser illumination, with no pathologic effects on DNA, helps to repair DNA after partial destruction by, for instance, external pathogens. ${ }^{19}$

The versatility of the pulsed IR LILI bio-modulatory action (wavelength $890 \mathrm{~nm}$, power 5-7 W, frequency $80 \mathrm{~Hz}$, exposure 2 minutes) has primarily manifested when simultaneous illumination has been applied to areas of skin lesions (rash) and carotid bulbs, ${ }^{20}$ providing potent prevention and treatment effects on the glandular, neuroendocrine, and immune systems. LLLT in patients with HSV-1 signs in the facial area results in the abortive disease course and acceleration of lesions healing in 92\% of cases. In addition, there have been observed significant reductions of the relapse rate - nearly by $75 \%$. For the prevention purpose, it is recommended to perform laser illumination of the mentioned areas every 2-3 months (3-4 procedures daily or every other day). ${ }^{7}$ Most often comprehensive treatment approaches combining different therapy modalities are applied.

In herpetic eruptions, Semenova and Vlasova ${ }^{21}$ used a continuous red spectrum LILI (wavelength $633 \mathrm{~nm}$, power density (PD) $2.5 \mathrm{~mW} / \mathrm{cm}^{2}$, exposure 6-8 minutes per lesion with 25-30 procedures per treatment course) as monotherapy or in combination with antiherpetic vaccine injection. Additionally, illumination on the projections of the spinal cord segments that innervate the impaired skin areas and maintaining treatment courses with 2-3 procedures per course were performed. A 2-4-fold reduction in relapse duration and a $2.5-5$-fold extension of the intercurrent period have been observed.

At the same time, Zimmermann ${ }^{22}$ has not achieved a significant therapeutic effect when using LILI in various forms of oral mucosa damages, including those caused by HSV-1, although the majority of patients accepted the LLLT positively. Unfortunately, the author has not described the procedure in detail, with no possibility of assessing the presented conclusions.

The most prominent therapeutic effect has been observed in patients with HSV who received comprehensive treatment with the use of Valtrex, LLLT in combination with Polyoxidonium, or psychotherapy. Recurrent occurrence decreased by more than three times, and the stable clinical remission duration reached up to $1.5-2$ years in 49 of 56 patients (85.7\%), compared to the groups of patients who received medication therapy only. The positive clinical treatment results are followed by a significant increase of the level of cells with a CD4 phenotype, the normalization of the immunoregulatory index $\mathrm{CD} 4 / \mathrm{CD} 8$, an increase in the proliferative and 
cytotoxic activity of T-lymphocytes, an improvement in the functional state of neutrophils, and the activation of interferon (IFN) production. The LLLT procedures were performed daily, with the use of the continuous red spectrum LILI in "BIO" mode, as well as pulsed IR LILI (wavelength $890 \mathrm{~nm}$, frequency 1000-2000 Hz, PD $0.3-0.4 \mathrm{~W} / \mathrm{cm}^{2}$ with an exposure of 20 seconds per skin lesion). The course treatment included 8-10 procedures, depending on the duration of the pathological process; the indication of LLLT administration was herpes of moderate severity. ${ }^{23}$

Non-invasive (percutaneous) laser blood illumination (NLBI) (wavelength $633 \mathrm{~nm}$, PD $20 \mathrm{~mW} / \mathrm{cm}^{2}$, and exposure 20-30 minutes on the cubital vein area) is highly effective when combined with immunomodulatory drugs. The aforementioned treatment regime promotes accelerated epithelization, as well as an improvement in local and general HVI-signs, along with a 2.5-fold reduction in instances of neuralgia of various localization, and a 3- to 4-fold extension of the remission period. Stimulating effects of laser blood illumination (in both in vitro and in vivo settings) on the parameters of humoral and cell-mediated immunity, as well as on non-specific defense factors, have been demonstrated. ${ }^{24}$ It should be noted that NLBI procedures require using pulsed LILI only and illuminating the projections of major vessels for not more than 5 minutes. ${ }^{16}$

Results yielded by randomized placebo-controlled, double-blind studies indicate that statistically significantly $(P<0.0001)$ high efficacy of the continuous LILI (wavelength $690 \mathrm{~nm}$, power $80 \mathrm{~mW}$, PD $80 \mathrm{~mW} /$ $\mathrm{cm}^{2}$, exposure time 10 minutes) can be attained on the herpes simplex lesions in the perioral area. All the patients involved in these trials were previously treated by oral administration of acyclovir $(800 \mathrm{mg} / \mathrm{d})$, with no visible effects. After the course of LLLT, the therapeutic effect was achieved in all 25 patients from the treatment group, with the remission lasting from 20 to 52 weeks, while in the placebo group remissions up to 20 weeks were observed in only three patients. ${ }^{25}$ In another randomized controlled trial (RCT) involving 232 patients in the treatment group and 322 subjects in the control group, the treatment group was exposed to illumination by continuous red spectrum LILI (wavelength $670 \mathrm{~nm}$, power $40 \mathrm{~mW}, \mathrm{PD}$ $51 \mathrm{~mW} / \mathrm{cm}^{2}$ ), whereby the exposure varied from 30-40 seconds on the vesicle areas at Stage I, to 94 seconds on the scab areas at Stage II, and finally to 20 seconds on the $\mathrm{C}_{2}-\mathrm{C}_{3}$ areas. The key outcome was an extension of the intercurrent period for up to three years. ${ }^{26}$

The continuous IR LILI procedure is less common. Nonetheless, good results have been demonstrated in the treatment of children with herpes simplex (wavelength $780 \mathrm{~nm}$, power $70 \mathrm{~mW}$, PD $62.5 \mathrm{~mW} / \mathrm{cm}^{2}$, exposure 80 seconds on each of four lesions), ${ }^{27}$ as well as in adults. ${ }^{28}$ Moreover, according to the available RCT data, the latency period can increase from 4 to 37.5 weeks after an LLLT course based on similar parameters. ${ }^{29}$

The issues stemming from high variability in the laser illumination parameters, lack of understanding of their optimization strategies, as well as lack of objective assessment criteria for treatment results in herpes labialis, were convincingly demonstrated in a recent systematic review. $^{30}$ Even though high LLLT efficacy has been confirmed in several trials, in some studies that fully adhered to the evidentiary standards, suboptimal laser illumination parameters were adopted. A continuous red spectrum and IR LILI were applied locally at wavelengths of $633,670,690,780$, and $870 \mathrm{~nm}$. Attempts to "standardize" the terminology and techniques have failed $^{31,32}$ since they did not take into account the mechanisms of bio-modulatory action (BA) of LILI or methodological aspects of LLLT.

Pathogenesis of genital herpes cannot be distinguished from that of any other HVI form caused by HSV-1 or HSV-2. Nonetheless, HSV-1 strains are isolated more often in the presence of skin lesions located on the face and upper extremities, whereas HSV-2 strains are more prevalent in genital lesions, but no direct correlations have been observed between antigen specificity and clinical localization of herpetic lesions. ${ }^{5,33}$ It is noteworthy that a number of experts distinguish this disease in females as a special category due to the immune system and neuro-endocrine regulatory mechanism peculiarities. In these cases, non-specific homeostasis maintenance and recovery methods are typically adopted, among which LILI is one of the most efficient and safe. ${ }^{34-37}$

In extant studies, positive dynamics of pathological processes were observed as early as after the third procedure performed using NLBI, with the patients reporting reduced itching and burning and sleep normalization (on days 5-6 in the control group). Moreover, in $77.8 \%$ of patients, the formed crusts fell off on days 5-6 (while the same outcome was achieved on days 10-12 in the control group). The remission period was extended by 1.5-2 times relative to the control group. Significant improvement was achieved in $50 \%$ of patients (4\% in the control group), while marked improvement was noted in $44.4 \%$ (64\% in the control group), and lack of dynamics was observed in only $5.6 \%$ (32\% in the control group). Nevertheless, the normalization of numerous cell-mediated and humoral immunity parameters was not achieved, resulting in the genital herpes relapse after 3-6 months. ${ }^{38}$ These results again demonstrate the need for LLLT procedure optimization (as noted previously, for NLBI, exposure should not exceed $5 \mathrm{~min}$, and the use of combined methods is recommended).

The clinical-immunological evaluation performed by Mamedova et $\mathrm{al}^{33}$ in 61 females with severe recurrent genital herpes infection demonstrates that the antibodies against HSV-1 were detected in $87.5 \%$ of the females, and 
the antibodies against HSV-2 were found in $72 \%$ of the cases; furthermore, $69.5 \%$ of the females had the antibodies against both types of viruses. Taking the inefficiency of the previous therapy into consideration, 30 females were assigned to the course of the intravenous laser blood illumination (wavelength $633 \mathrm{~nm}$, power $1 \mathrm{~mW}$ ), and 31 females were assigned to take the immunomodulators additionally to the LLLT. The treatment was conducted from the fifth or sixth day of the menstrual cycle once a day or every other day, with 7 procedures per treatment course. The exposure time during the first five procedures was $15 \mathrm{~min}$, with its subsequent extension up to 30 minutes.

The analysis of the dynamics of peripheral immunity parameters after the LLLT treatment course revealed a significant increase in the relative number of CD8positive cells compared to that before the treatment. The following administration of the IFN-inducers resulted in a statistically significant increase in $\mathrm{CD} 4^{+}-T$ - и $\mathrm{CD} 19^{+}$-Blymphocytes content. After the comprehensive treatment with the use of intravenous laser blood irradiation (ILBI), a significant decrease in female proportion with a low content of NK-cells (which exhibit cytotoxic activity against viruses) from $37 \%$ to $8 \%$ was observed. Thus, the comprehensive treatment with the use of ILBI positively impacted on the immune system by normalizing the content of peripheral blood lymphocytes of different phenotypes in females with severe genital herpesvirus infection. After the LLLT treatment, the serum IFN-levels decreased, compared to baseline values, and reached the levels in the control group. After the course of LLLT, the production of the IFN- $\alpha$ and IFN- $\gamma$ induced in the leucocytes statistically significantly differed from the levels before the treatment. Mean values of all the proinflammatory cytokines levels in the cervical mucus of females with genital herpes virus infection decreased nearly two-fold compared to the baseline after the course of comprehensive therapy. Stable remission after ILBI treatment was observed in $19 \%$ of the females with HSV, and less frequent and mild clinical relapses were observed in $64 \%$ of the females. The recurrent occurrence rate was significantly reduced by 2 times compared to that before the treatment. In the presence of relapse that occurred after the course of comprehensive therapy, a significant reduction of herpetic lesions, improvement of symptoms, and shortening of relapse resolution time, compared to the period before the treatment, were observed. In the follow-up period, 16 females became pregnant during 36 months, 14 of them were diagnosed with o primary or secondary infertility (47\%), with no contraceptives use. $^{33,39}$

Comparative analysis of the dynamics of immunological parameters after the course of comprehensive therapy in females with the use of laser ultraviolet blood illumination (LUVBI) (wavelength $365 \mathrm{~nm}$, power 2-3 mW, exposure- time 8 min with 6 procedures per course), at 6 and 12 months after the course treatment completion, revealed significant positive changes in immunological parameters of the peripheral blood: recovery of the quantitative and sub-population leucocyte content and normalizing the intercellular relations of T-lymphocyte populations, namely an increase in the number of relative $\mathrm{CD}^{+}-$ cells, an increase in the immunoregulatory index, and an increase in the number of relative and absolute $\mathrm{CD} 4^{+}$-, CD3-CD16 ${ }^{+}$, CD3-CD16 ${ }^{+}$-CD3+HLA-DR ${ }^{+}$-cells. The latter effect resulted in an increase in the number of immunocompetent cells in the peripheral blood, the normalization of the growth of absorption capacity of neutrophils (by latex-test), the recovery of the biocide functions of the neutrophils (by nitro blue tetrazolium (NBT)-test), functional reserves, the serum levels of $\operatorname{IgA}$ and $\operatorname{IgM}$, and the blood level of IFN-gamma. When studying the phagocytic activity and neutrophil activity in NBT-test by the cells' abilities to absorb the latex microspheres and to recover nitro blue tetrazolium, the reduction of the studied parameters before treatment and their normalization after the course of comprehensive therapy have been observed. ${ }^{40}$

Thus, ILBI application in the treatment of patients with genital herpes results in complete or partial recovery of the qualitative and quantitative peripheral neutrophils content, their absorptive capacity and oxygen-dependent metabolism, expressed in the increase in the activity of peripheral blood phagocytes by their ability to capture latex particles and to produce reactive oxygen species. The aforementioned production results in the increase of phagocytic activity of neutrophils by $16 \%$, the increase of phagocytic intensity of neutrophils by $38 \%$, the enhancement of spontaneous NBT-test activity by $34 \%$, the enhancement of spontaneous NBT-test intensity by $19 \%$, the growth of the peripheral blood neutrophils functional reserve by $26 \%$, the increase of IFN- $\gamma$ content by $87 \%$, and the increase of IgA content by $29 \%$. The identified positive dynamics of immunological parameters reflects the restored potential of innate and acquired immunity factors in females, who received comprehensive therapy with the use of intravenous laser blood illumination by UV LILI. ${ }^{40}$

In pregnant women, HVIs hold a special place among the causes of perinatal morbidity and mortality. In some cases, these infections may determine children's health in the first years of life and even cause their disability. Acute (primary) herpesvirus infections, regardless of the relatively low incidence in pregnant women, are generally complicated with the development of primary placental insufficiency, blasto- and embryo-fetopathies, fetal loss, or development of severe congenital infections with central nervous system impairment. Persistent (not primary) infections are linked with non-specific body resistance disorders and the formation of the autoimmune 
inflammatory process component. In the presence of gestation immunity, this leads to antibody persistence, development of hemostatic homeostasis disorders, fetoplacental insufficiency, and intrauterine infection. ${ }^{41}$

Chronic HSV and CMV infections in pregnant women result in adaptive-regulatory mechanism impairment which includes a set of changes in the immune and neuroendocrine systems, hemostasis disorder, dysfunction of the natural detoxication systems, central and peripheral hemodynamics disorders, and tissue respiration damage, resulting in complicated gestation courses and delivery. Infections are characterized by the development of early toxicosis $(52.2 \%, 36.1 \%$, and $52 \%)$ and late gestosis $(21.7 \%$, $23.4 \%$, and $24 \%)$, threatened abortion in the I $(26 \%$, $19.1 \%$ and $20 \%)$ and II $(13 \%, 13 \%$ and $16 \%)$ gestation trimesters, intrauterine fetus hypoxia $(13 \%, 23.4 \%$, and $12 \%)$, intrauterine growth restriction $(4.3,6.4$, and $4 \%)$, and premature birth $(39.1 \%, 31.9 \%$, and $40 \%){ }^{42,43}$

LLLT, particularly ILBI, has successfully been used for more than 30 years at the Academician V.I. Kulakov National Medical Research Center of Obstetrics, Gynecology and Perinatology of the Ministry of Health of Russia. Results obtained from numerous studies conducted by the institution staff have become the basis for the development of clinical recommendations and teaching guides for physicians. ${ }^{44}$ It was observed that ILBI635 (wavelength $635 \mathrm{~nm}$, power 2-3 mW, exposure 15-20 minutes) when applied in pregnant women with viral infections after 32 weeks of gestation allowed achieving remission of a viral infection, prolonging pregnancy, and reducing the percentage of infectious complications in newborns by $25 \%$ The remission duration after the course of LLLT was not less than 4-4.5 months. ${ }^{45}$

The use of comprehensive LLLT in recurrent HSV infections is more efficient than the use of medication therapy only. This approach offers the direct positive effect of the achievement of stable remission of the infectious disease in $95 \%$ of pregnant women, the increase of the immunoregulatory index by an average of 1.5 times compared to baseline values, clinical recovery acceleration (by $3.4 \pm 1.1$ days), and the prevention of recurrence. ${ }^{42}$

ILBI stimulates cell-mediated and humoral immunity, as well as non-specific defense responses in pregnant women with HSV and CMV infections. The application of the ILBI provides the reduction of the increased CIC-levels by 1.5 times, the IgM levels by $23 \%$, and the IgE level by $34 \%$, whereby the titers of antibodies against opportunistic microorganisms decrease by 1.5 times and the titers of antibodies against gram-negative bacteria endotoxins by 2 times compared to baseline parameters. In addition, the significant increase of the T-helper component (CD4) of lymphocytes, the normalization of T-cells (CD3), suppressor T-cells (CD8), and B (CD19) lymphocytes values, as well as the increase of the immunoregulatory index up to $1.7 \pm 2.6$ have been observed. The therapy also ensures the stabilization of the coagulation parameters due to the plasma and platelet components of hemostasis. The LLLT is followed by the normalization of thromboelastogram parameters. The most effective option is the combined use of plasmapheresis and ILBI; in this case, both methods have no negative impact on the fetoplacental complex that is confirmed by the increase of the initially decreased (by 1.2-1.5 times) levels of the placental lactogen, progesterone, and cortisol. ${ }^{43,46}$

The study performed by Chernova ${ }^{47}$ showed high efficacy of the comprehensive treatment, which included multiple procedures of laser illumination in females with persistent CMVI and urogenital tract infections. The course of the comprehensive LLLT was followed by the normalization of urogenital microbiota in $88.89 \%$ of the patients, as well as by the normalization of the subpopulation lymphocyte content, neutrophils phagocytosis parameters, and IFN status in serum and cervical-vaginal mucus, which facilitated the CMVI conversion into the latent state in $91.04 \%$ of the females. The combined LLLT procedure was developed for patients with CMVI, using the different spectral ranges of LILI. The course involves 15 ILBI procedures concomitantly with antiviral therapy, alternating the regimes every other day: on the first day LUVBI (wavelength $365 \mathrm{~nm}$, power $2 \mathrm{~mW}$, exposure 2 minutes) is performed, on the second day - ILBI-525 (green spectrum, wavelength $525 \mathrm{~nm}$, power $2 \mathrm{~mW}$, exposure 8 minutes), on the third day LUVBI again, and so on. The combined LLLT method terminates reactivation of cytomegalovirus infection in the urogenital tract, shortens the duration of subsequent recurrent, extends intercurrent periods, and helps to convert a viral infectious process into a persistent state due to the immune response activation and IFN status normalization, with the concomitant suppression of hyperactive processes. ${ }^{48}$

Even in the 1980s of the last century, when LLLT strategy was arousing mistrust abroad, a sufficient number of articles were published, demonstrating that the LLLT facilitated the acceleration of skin lesion resolution, the reduction of disease severity, and the improvement of pain syndrome and symptoms of postherpetic neuralgia. ${ }^{49-55}$ Kemmotsu et al, ${ }^{51}$ when illuminating shingles lesions with helium-neon laser in continuous mode (wavelength $633 \mathrm{~nm}$, power 8-9 $\mathrm{mW}, \mathrm{PD} 25-30 \mathrm{~mW} / \mathrm{cm}^{2}$, exposuretime 5 minutes, 5-20 procedures), noted the significant improvement of pain syndrome and the acceleration of skin lesions healing. These parameters have become most often used in practice.

The continuous diode IR-lasers also were commonly used (wavelength $830 \mathrm{~nm}$ ). In the double-blind randomized cross-over trial, Moore et $\mathrm{al}^{56}$ presented data from more than 9-year experience in the treatment of hundreds of patients who suffered from postherpetic neuralgia, using IR LILI (power: $60 \mathrm{~mW}$, point-contact 
scheme). During the treatment, the pain level was reduced by more than $50 \%$ in $85 \%$ of the patients. The most prominent therapeutic effect was observed with lesions located in the chest area (pain level reduction by $78 \%$ and relapse rate reduction by $22 \%$ ), with lesions located in the head area pain decreased by $61 \%$ and relapse rate decreased by $33 \%$. The application of laser illumination in the acute phase of shingles resulted in postherpetic neuralgia occurrence reduction; the treatment of the patients with cancers was also highly efficient. ${ }^{53,54,57}$ The studies with a double-blind control design demonstrated that in postherpetic neuralgia treatment with the use of continuous IR LILI (wavelength $-830 \mathrm{~nm}$ ), the power of $150 \mathrm{~mW}$ is more effective than $60 \mathrm{~mW} .{ }^{58}$ It was shown that LLLT, both in HSV-1, HSV-2 and in shingles, is essentially effective at early stages of disease (more than 10, 000 procedures performed). ${ }^{59}$

More than one-third of physiotherapists in Northern Ireland pointed out the efficacy of LLLT, considering it more preferable compared to other physiotherapy methods (interference currents electrotherapy, pulsed electromagnetic fields, short-wave diathermy, ultrasound).$^{60}$

The corresponding studies were also conducted in Russia, where neurologists are well familiar with LLLT effects in shingles treatment. According to the data reported by Elkin et al, ${ }^{61}$ the continuous laser light of red spectrum $(633 \mathrm{~nm})$ on skin lesions located on the facial, chest, lumbar and gluteal areas was effective in all patients. Clinical effect was observed after four or five procedures performed as a daily regime; complete rash resolution was achieved 7 to 12 days from the start of treatment. The best results were observed with the lesions located on the face. The use of topical antiviral medications (IFN, oxolin, and tebrofen ointments and deoxyribonuclease solution), in combination with antiviral drugs and laser illumination on lesion areas (wavelength $633 \mathrm{~nm}$, continuous mode, $\mathrm{PD} 20 \mathrm{~mW} / \mathrm{cm}^{2}$, exposure-time 10 minutes), in patients with trigeminal neuralgia infected by HSV-3 resulted in the resolution of the pathological process and deflorescence five to six days earlier than without laser treatment application. ${ }^{62}$ For more extended remission, it is recommended to perform block anaesthesia of the corresponding trigeminal nerve branch. ${ }^{63}$

\section{Conclusion}

Even from this brief literature review, it can confidently be concluded that LLLT is a potential prospective treatment modality for patients infected with the herpesvirus. However, there is an urgent need to improve the methodologies of laser action and to optimize the combination of methods with the use of current medications. Unfortunately, according to the analysis of published reports, the laser illumination parameters used in the majority of studies were too far from the optimal and effective ones, although it is clear that we should be guided by the rules that have been established by clinical recommendations and proved over time. ${ }^{64}$

The key question regarding the practical implementation of any treatment method is to understand the rules for its use. It is well known that combined LLLT methodologies are the most efficient; however, the optimization of their parameters is nearly always individually tailored. For instance, when using LLLT concomitantly with pharmaceuticals, the most common questions are about drug dosage form and route of administration. According to a number of reports, with the presence of a skin rash, exposure of LILI on the site with applied antiviral ointment (active substance - acyclovir) ${ }^{65,66}$ is the most effective method. In contrast, results from the RCT, which included 60 subjects over 16 years old, show that, irrespective of virus type (HSV-1 or HSV-2) and lesion localization (facial or genital area), the clinical efficacy of either laser illumination (wavelength $633 \mathrm{~nm}$, power $20 \mathrm{~mW}$ ) locally or acyclovir orally was not different. But the best results could be achieved using a combination of both methods. ${ }^{67}$

For initial procedures, the optimal exposure time is within the first 24 hours of the onset of virus reactivation. However, in the case of laser application, on the second day after infection, only acyclovir "works"; that is, laser light no longer has a potentiating effect, whereas if illumination is started during the first four hours, only laser light will ensure a reliable result with no medication use. $^{68}$

Thus, the literature data and our studies show that for any variants of herpesvirus infection, it is most effective to combine local laser illumination of lesions by continuous LILI of the red spectrum (wavelength 635 $\mathrm{nm}$, power density $15-25 \mathrm{~mW} / \mathrm{cm}^{2}$, exposure 5 minutes) with concomitant application of gels (creams) with an antiviral drug to this area. The technique is well known in Russia as laser phoresis that is an enhancement of the penetration of a biologically active substance after illumination with LILI. ${ }^{69}$ But local illumination must be supplemented with the combined method of intravenous laser blood illumination LUVBI + ILBI-525, which are carried out on the same day. However, the ILBI options themselves alternate, on the first day - LUVBI (wavelength $365 \mathrm{~nm}$, power $2 \mathrm{~mW}$ at the fiber output, exposure 3-5 minutes), on the next day - ILBI-525 (wavelength 525 $\mathrm{nm}$, power $2 \mathrm{~mW}$ at the output of the fiber, exposure 7-10 minutes), and so on. ${ }^{48}$ In total there are up to 10-12 daily procedures for the course.

In future research, it is necessary to clarify aspects of the combination of LLLT with drugs and assess the duration of remission.

\section{Author's Contribution}

The author contributed solely to the work. 


\section{Ethical Considerations}

Not applicable.

\section{Conflict of Interests}

The authors declare no conflict of interest.

\section{Financial Support}

No competing financial interests exist.

\section{References}

1. Djudjun AD, Polion NN, Nagornyj AE. Herpesvirus infection. Clinical and immunological features. Clinical lecture. Derm Kosm Seksop. 2015;3-4:119-42. [Russian].

2. Ptaszyńska-Sarosiek I, Dunaj J, Zajkowska A, Niemcunowicz-Janica A, Król M, Pancewicz S, et al. Postmortem detection of six human herpesviruses (HSV-1, HSV-2, VZV, EBV, CMV, HHV-6) in trigeminal and facial nerve ganglia by PCR. PeerJ. 2019;6:e6095. doi: 10.7717/ peerj.6095.

3. Shhubelko RV, Zujkova IN, Shul'zhenko AE. Human herpesvirus infections: clinical features and treatment options. RMJ. 2018;8(I):39-45. [Russian].

4. Chernova NI. Cytomegalovirus infection in women with infectious diseases of the urogenital tract. Clinic, diagnosis, treatment [dissertation]. Moscow; 2014. [Russian] https:// search.rsl.ru/ru/record/01005102483.

5. Semenova TB. Genital herpes in women. RMJ. 2001;9(6):237-42. [Russian]

6. Boeckh M, Corey L. Adoptive immunotherapy of viral infections: should infectious disease embrace cellular immunotherapy? J Infect Dis. 2017;216(8):926-8. doi: 10.1093/infdis/jix360.

7. Prohonchukov AA, Zhizhina NA, Banchenko GV. Prevention and treatment of facial herpes with the help of the optical laser optical device Optodan. Stomatologija. 2006;3:78-82. [Russian]

8. Proceedings of the All-Union Seminar on the exchange of experience in the introduction of helium-neon lasers for the treatment of diseases of the oral mucosa and periodontal disease. Moscow; 1979. https://search.rsl.ru/ ru/record/01007470736. [Russian]

9. de Freitas PM, Simões A. Lasers in Dentistry: Guide for Clinical Practice. John Wiley \& Sons, Inc; 2015. doi: 10.1002/9781118987742.

10. Shakarian AA. The results of the use of helium-neon laser in the treatment of herpetiform keratitis in children. Abstracts Int. conf. "New in laser medicine and surgery. Part 2. Pereslavl'-Zalesskij, 1990. https://search.rsl.ru/ru/ record/01001565838. [Russian]

11. Karmal'kova EA. Treatment of acute herpetic stomatitis in children using helium-neon laser [dissertation]. Minsk; 1991. https://search.rsl.ru/ru/record/01000044706. [Russian].

12. Kamalova MK. Use of laser therapy in the treatment of chronic recurrent herpetic stomatitis for children. Eur Sci Rev. 2018;7-8:120-1.

13. Bello-Silva MS, de Freitas PM, Aranha AC, Lage-Marques
JL, Simões A, de Paula Eduardo C. Low- and high-intensity lasers in the treatment of herpes simplex virus 1 infection. Photomed Laser Surg. 2010;28(1):135-9. doi: 10.1089/ pho.2008.2458.

14. Cernavin I. Low-level laser therapy in the treatment of herpes labialis. Laser. 2010;1:16-7.

15. Ferreira DC, Martins FO, Romanos MT. Impact of lowintensity laser on the suppression of infections caused by Herpes simplex viruses 1 and 2: in vitro study. Rev Soc Bras Med Trop. 2009;42(1):82-5. doi: 10.1590/s003786822009000100018.

16. Ferreira DC, Reis HL, Cavalcante FS, Santos KR, Passos MR. Recurrent herpes simplex infections: laser therapy as a potential tool for long-term successful treatment. Rev Soc Bras Med Trop. 2011;44(3):397-9. doi: 10.1590/s003786822011000300029.

17. Perrin D, Jolivald JR, Triki H, Garbarg-Chenon A, Lamotte D'Incamps B, Lefevre B, et al. Effect of laser irradiation on latency of herpes simplex virus in a mouse model. Pathol Biol (Paris). 1997;45(1):24-7.

18. Saleh HM, Shaker AS, Saafan AM, Ibrahim AK. Herpes virus reactivation by low-intensity diode and $\mathrm{CO}_{2}$ lasers. Photomed Laser Surg. 2011;29(2):83-90. doi: 10.1089/ pho.2009.2744.

19. da Silva Sergio LP, da Silva Marciano R, Teixeira GR, da Silva Canuto K, Polignano GA, Guimarães OR, et al. Therapeutic low-intensity red laser for herpes labialis on plasmid survival and bacterial transformation. Photochem Photobiol Sci. 2013;12(5):930-5. doi: 10.1039/c3pp25394e.

20. Patent 2101046 RU, MPK A61N5/06. A method for the treatment of inflammatory purulent destructive processes of the maxillofacial area and neck. No. 94031063/14; 23.08.1994; publ. 10.01.1998. https://www.fips.ru/registersdoc-view/fips_servlet?DB=RUPAT \&DocNumber $=$ 2101046\&TypeFile=html. [Russian]

21. Semenova TB, Vlasova PI. Treatment of simple recurrent skin herpes with the help of helium-neon laser rays. In: Abstracts All-Union conference on the use of lasers in medicine. Moscow; 1984. https://search.rsl.ru/ru/ record/01001193793. [Russian].

22. Zimmermann M. [Studies on the therapeutic efficacy of a HeNe laser]. Dtsch Z Mund Kiefer Gesichtschir. 1990;14(4):313-9. [German].

23. Karimova M. Psycho-emotional, clinical and immunological features and a comprehensive method for the treatment of recurrent herpes [dissertation]. Moscow; 2001. https://emll.ru/find?iddb=17\&ID=RUCMLBIBL-0000696123. [Russian]

24. Kolieva MH. The use of laser irradiation of blood, mielopid and poludana in the complex therapy of patients with recurrent forms of herpes simplex (clinical and immunological study) [dissertation]. Moscow; 1995. https://search.rsl.ru/ru/record/01000077400. [Russian].

25. Schindl A, Neumann R. Low-intensity laser therapy is an effective treatment for recurrent herpes simplex infection. Results from a randomized double-blind placebocontrolled study. J Invest Dermatol. 1999;113(2):221-3. doi: 10.1046/j.1523-1747.1999.00684.x.

26. Muñoz Sanchez PJ, Capote Femenías JL, Díaz Tejeda A, Tunér J. The effect of 670-nm low laser therapy on herpes 
simplex type 1. Photomed Laser Surg. 2012;30(1):37-40. doi: 10.1089/pho.2011.3076.

27. Stona P, da Silva Viana E, Dos Santos Pires L, Blessmann Weber JB, Floriani Kramer P. Recurrent labial herpes simplex in pediatric dentistry: low-level laser therapy as a treatment option. Int J Clin Pediatr Dent. 2014;7(2):140-3. doi: 10.5005/jp-journals-10005-1252.

28. de Carvalho RR, de Paula Eduardo F, Ramalho KM, Antunes JL, Bezinelli LM, de Magalhães MH, et al. Effect of laser phototherapy on recurring herpes labialis prevention: an in vivo study. Lasers Med Sci. 2010;25(3):397-402. doi: 10.1007/s10103-009-0717-9.

29. Lacour J. [Low-power laser and recurrent labial herpes]. Ann Dermatol Venereol. 2000;127(6-7):652-6. [French]

30. Al-Maweri SA, Kalakonda B, AlAizari NA, Al-Soneidar WA, Ashraf S, Abdulrab S, et al. Efficacy of low-level laser therapy in management of recurrent herpes labialis: a systematic review. Lasers Med Sci. 2018;33(7):1423-30. doi: 10.1007/s10103-018-2542-5.

31. de Paula Eduardo C, Aranha AC, Simões A, Bello-Silva MS, Ramalho KM, Esteves-Oliveira M, et al. Laser treatment of recurrent herpes labialis: a literature review. Lasers Med Sci. 2014;29(4):1517-29. doi: 10.1007/s10103-013-1311-8.

32. de Paula Eduardo C, Bezinelli LM, de Paula Eduardo F, da Graça Lopes RM, Ramalho KM, Bello-Silva MS, et al. Prevention of recurrent herpes labialis outbreaks through low-intensity laser therapy: a clinical protocol with 3-year follow-up. Lasers Med Sci. 2012;27(5):1077-83. doi: 10.1007/s10103-011-1019-6.

33. Mamedova SJ, Fedorova TA, Van'ko LV, Ochan AS. Intravascular laser irradiation of blood in the treatment of genital herpes. Proceedings of scientific and practical. conf. "Medical ozone and quantum hemotherapy in obstetric and gynecological practice. Moscow; 2007. https://www. critical.ru/news/2007_11_16_lections.htm. [Russian].

34. Serov VN, Tsahilova SG, Bonarcev PD, et al. Structural and functional state of peripheral blood components in women with a typical form of genital herpes during endovascular laser irradiation of blood. Russian Association of Obstetricians and Gynecologist. 1998;1:17-21. https:// emll.ru/find?iddb=17\&ID=RUCML-BIBL-0000411521 [Russian]

35. Serov VN, Cahilova SG, Bonarcev PD. Structural and functional state of peripheral blood components in women with a typical form of genital herpes during endovascular laser irradiation of blood. Venerolog. 2004;11:44-8. https:// emll.ru/find?iddb=17\&ID=RUCML-BIBL-0001277991. [Russian].

36. Chernova NI. The state of systemic immunity in women of reproductive age with a cytomegalovirus infection of the urogenital tract. Russian Bulletin of the ObstetricianGynecologist. 2014;14(2):23-8. [Russian].

37. Chernova N, Perlamutrov YN. Cytomegalovirus infection of the urogenital tract in women. Russian Journal of Skin and Venereal Diseases. 2014;17(2):54-60. doi: 10.17816/ dv36893. [Russian].

38. Dzhegutanov KE. The effectiveness of complex, staged treatment of patients with genital herpes [abstract of the dissertation]. Moscow; 1996. https://emll.ru/ find?iddb=17\&ID=RUCML-BIBL-0000413537. [Russian].
39. Mamedova SJu. Intravascular laser radiation in the treatment of genital herpes. J New Med Tech. 2007;14(4):613. doi: 10.24411/2075-4094-2019-16467. [Russian].

40. Gizinger OA, Ziganshin OR, Shemetova MA. Analysis of immunomodulating effects of low-intensity laser in the treatment of herpes viral infections of the urogenital system. Russimmun J. 2016;10(19):426-8. https://www. lazmik.ru/assets/files/93/2016_6-8.pdf. [Russian].

41. Fedorova E, Zarochenceva NV, Polesko IV, Malinovskaja VV. Etiopathogenesis of spontaneous abortion: modern concepts. Vopr Ginekol Akush Perinatol. 2015;1(2):538. [Russian]. https://www.phdynasty.ru/upload/ medialibrary/ 9f4/9f4b684aa80c2d14033120b9fb00c24f. pdf.

42. Zueva JeA. Effect of HSV infection on course of gestation and fetal condition: method of comprehensive treatment of relapsing HSV infection [dissertation]. Sankt-Peterburg; 1996. https://emll.ru/find?iddb=17\&ID=RUCMLBIBL-0000340528. [Russian].

43. Tsakhilova SG. Plasmapheresis and blood photomodification in the treatment of pregnant women with viral infection [abstract of the dissertation]. Moscow; 1999. https://emll.ru/find?iddb=17\&ID=RUCMLBIBL-0000483629. [Russian].

44. Kulakov VI, Serov VN, Abubakirova AM, Fedorova TA. Intensive therapy in obstetrics and gynecology (efferent methods). Moscow: MIA; 1998. https://emll.ru/ find?iddb=17\&ID=RUCML-BIBL-0000409864. [Russian]

45. Kulakov VI, Serov VN, Abubakirova AM, et al. Non-drug method in the prevention and treatment of purulent-septic complications in obstetrics, gynecology and neonatology: A Handbook for Doctors. Moscow; 2002. [Russian]

46. Kisselev SB, Moskvin SV. The Use of Laser Therapy for Patients with Fibromyalgia: A Critical Literary Review. $J$ Lasers Med Sci. 2019;10(1):12-20. doi:10.15171/ jlms.2019.02.

47. Chernova NI. Cytomegalovirus infection in women with infectious diseases of the urogenital tract. Clinic, diagnosis, treatment. [abstract of the dissertation]. Moscow; 2015. https://lazmik.ru/assets/files/625/Chernova_2015.pdf. [Russian].

48. Patent 2513474 RU, MPK A61N5/067. A method for treating a reactive form of a cytomegalovirus infection of the urogenital tract in women of reproductive age. No. 2013115641/13. 08.04.2013. publ.17.02.2014. https://new. fips.ru/registers-doc-view/fips_servlet?DB=RUPAT\&Doc Number $=2513474 \&$ TypeFile $=$ html. [Russian].

49. Dionetto P, D’Ovido M, Franz S. Treatment of Herpes Zoster with LLLT and magnetotherapy. Laser Ther. 1994;6(1):35.

50. Farago K. Low power laser in dermatology. Laser Ther. 1994;6(1):43

51. Kemmotsu O, Saito Y, Enya T. He-Ne-laser irradiation accelerates healing and reduces pain in the acute phase of Herpes Zoster. Laser Ther. 1994;6(1):44.

52. Matsumura C, Ishikawa F, Imai M, Kemmotsu O. Useful effect of application of helium-neon LLLT on an early stage case of Herpes Zoster: a case report. Laser Ther. 1993;5(1):43-6. doi: 10.5978/islsm.93-CR-01.

53. Moore KC. Laser therapy in post herpetic neuralgia. Laser 
Ther. 1996;8(1):48.

54. Moore KC. Postherpetic neuralgia as a complication of malignant disease and its treatment using a GaAlAs diode laser. Laser Ther. 1996;8(1):49.

55. Otsuka H, Numazawa R, Okubo K, Enya T, Saito Y, Kemmotsu O. Effects of helium-neon laser therapy on Herpes Zoster pain. Laser Ther. 1995;7(1):27-32. doi: 10.5978/islsm.95-OR-05.

56. Moore KC, Hira N, Kumar PS, Jayakumar CS, Ohshiro T. A double blind crossover trial of low level laser therapy in the treatment of postherpetic neuralgia. Laser ther. 2004;14(2):61-4. doi: 10.5978/islsm.14.0_61.

57. Navarro R, Marquezan M, Cerqueira DF, Silveira BL, Corrêa MS. Low-level-laser therapy as an alternative treatment for primary herpes simplex infection: a case report. J Clin Pediatr Dent. 2007;31(4):225-8. doi: 10.17796/jcpd.31.4.yl4178275423171p.

58. Yamada H, Ogawa H. Comparative study of $60 \mathrm{~mW}$ diode laser therapy and $150 \mathrm{~mW}$ diode laser therapy in the treatment of postherpetic neuralgia. Laser Ther. 1995;7(2):71-4. doi: 10.5978/islsm.95-OR-10.

59. Rozsa G. Laser therapy and its combinid treatments in the first provincial laser center of Hungary. Laser Ther. 1994;6(1):69.

60. Baxter GD, Bell AJ, Allen JM, Ravey J. Low level laser therapy: current clinical practice in Northern Ireland. Physiotherapy. 1991;77(3):171-8. doi: 10.1016/s00319406(10)61696-3.

61. Elkin VD, Kljachin VM, P'jankov ZA. Experience of using low-intensity laser radiation for the treatment of shingles. Perm; 1986. [Russian].

62. Lukach ID, Sapronova LB, Sichevoj VP, Bezjazychnyj VI. Treatment of trigeminal neuralgia complicated by herpes zoster by laser therapy in combination with antiviral drugs. The use of lasers in science and technology. Novosibirsk; 1992. http://webirbis.spsl.nsc.ru/irbis64r_01/ cgi/cgiirbis_64.exe?P21DBN=CAT\&I21DBN=CAT_

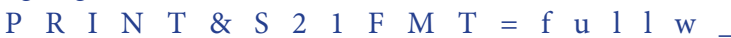

print\&C21COM=F\&Z21MFN=38590. [Russian].

63. Lukach ID, Sapronova LB, Sichevoj VP. Comparative efficacy of laser therapy using blockade of vitamin B-12 $500 \mathrm{mg}$ and a solution of trimekain 2\% with norepinephrine in the treatment of trigeminal neuralgia. The use of lasers in science and technology. Novosibirsk; 1992. http://webirbis.spsl.nsc.ru/irbis64r_01/cgi/ cgiirbis_64.exe?P21DBN=CAT\&I21 DBN =CAT_ PRINT\&S21FMT =fullw_print\&C21COM=F\&Z21MFN= 38590. [Russian].

64. Moskvin SV. Low-level laser therapy in Russia: history, science and practice. J Lasers Med Sci. 2017;8(2):56-65. doi: $10.15171 /$ jlms.2017.11.

65. Semenova TB. Laser therapy in the complex treatment of herpes. Laser Med. 1997;1(1):38-9. https://www.elibrary.ru/ download/elibrary_42371477_43543679. pdf. [Russian].

66. Brignardello-Petersen R. Treatment of lesions associated with herpes labialis with low level laser therapy may result in a decrease of pain and recovery time compared with acyclovir. J Am Dent Assoc. 2017;148(10):e153. doi: 10.1016/j.adaj.2017.07.009.

67. Velez-Gonzalez M, Urrea-Arbelaez A, Nicolas M, SerraBaldrich E, Perez J, Pavesi M, et al. Treatment of relapse in herpes simplex on labial and facial areas and of primary herpes simplex on genital areas and" area pudenda" with low-power He-Ne laser or Acyclovir administered orally. In: Effects of Low-Power Light on Biological Systems. Vol 2630. SPIE; 1996. doi: 10.1117/12.230040.

68. Votjakov VI, Sokolovskaja AD, Andreeva OD. On the issue of optimizing the combined effects of laser radiation and chemotherapy on the course of experimental herpes infection. In: Optimization of exposures in physiotherapy. Minsk: Belarus; 1980. https://emll.ru/ find?iddb=17\&ID=RUCML-BIBL-0001044086. [Russian].

69. Moskvin S, Konchugova TV. Justification of the use of laser phoresis of biologically active substances. Vopr Kurortol Fizioter Lech Fiz Kult. 2012;5:57-63. [Russian]. 